\title{
Time Resolved Tomographic PIV Measurements of Rough-Wall Turbulent Channel Flow
}

\author{
Rinaldo Miorini, Cao Zhang and Joseph Katz
}

\author{
Department of Mechanical Engineering, Johns Hopkins University, Baltimore, USA \\ 1katz@jhu.edu
}

\begin{abstract}
Rough-wall turbulent channel flow is investigated via time-resolved tomographic particle image velocimetry (TPIV). To facilitate 3D measurements in the bulk flow as well as very close to the rough surface the optical refractive index of the transparent acrylic channel wall is matched with that of the liquid, a concentrated solution of sodium iodide in water (62\%-64\% by weight). The roughness consists of staggered pyramidal elements with $h / k=55$ (half channel to roughness heights ratio) and $k^{+}=64$, which satisfies the "well-characterized" rough wall boundary layer conditions. The Reynolds number based on half channel height is 40000 . Our research is aimed at studying mechanisms involved with generation of coherent structures as large-scale outer layer turbulence and the mean flow interact with the roughness elements, and their subsequent propagation away from the wall. Data is acquired using four high speed cameras at 3000 frames per second, the sample volume size is $40.6 \times 44.1 \times 17.4 \mathrm{~mm}^{3}$, and the vector spacing after 3D cross-correlation with $75 \%$ overlap is $0.588 \times 0.588 \times 0.588 \mathrm{~mm}^{3}$. Consistent with recent observations based on holographic PIV measurements, the TPIV data confirms the formation of U-shaped vortices that wrap around the low speed regions above peaks of the pyramids. Interactions among quasi-streamwise "legs" of vortices generated by neighboring pyramids generate powerful ejection events that lift these structures away from the wall.
\end{abstract}

\section{INTRODUCTION}

The motion of a fluid in the "roughness sublayer" at turbulent regimes has been a topic of debate in the scientific community. Inside the roughness sublayer, which extends from the wall up to 2-5 times the roughness height $(k)$, viscous effects are non-negligible and the flow shows "imprints" of the roughness. Conversely, outside the layer one can ask whether the influence of roughness and viscosity diminishes, i.e. the Towsend's similarity hypothesis [1], as applied to rough wall cases, holds (Roupach et al., [2]). The special case of zero-pressure-gradient (ZPG) boundary layer flow over three-dimensional roughness has been studied extensively. For instance, Shockling et al. [3], Wu and Christensen [4], Schultz and Flack [5] and Flack et al. [6], along with measurements performed in our laboratory, e.g. Hong et al. [7], Hong et al.[8], and Talapatra and Katz [9,10] support the validity of the Taylor's hypothesis. On the contrary, Krogstad, and Antonia [11], Tachie et al. [12], Shah and Tachie [13], and Keirsbulck et al. [14] have observed significant changes to the Reynolds stresses that extend well into the outer layer for flows over woven mesh and transverse bar roughness. Jiménez [15] points out that most rough wall studies have involved low values of either the scale separation $\delta k^{1}$, where $\delta$ is the boundary layer thickness, or the equivalent sand roughness $\left(k_{\mathrm{s}}{ }^{+}=k u_{\tau} / v, u_{\tau}\right.$ is the friction velocity). In the former case, the flow can be better described as turbulence over obstacles, while in the latter flows are located in the transitionally rough regime. Typically, in order to obtain so-called a "well-characterized" boundary layer for testing Townsend's hypothesis, $k^{+}>50$ and $\delta k^{1}$, and their product $\left(\delta^{+}\right)$should be at least 4000 . Recent experiments by Shockling et al. [3], Wu and Christensen [4], Schultz and Flack [5], and Volino et al. [16] have shown that the wall similarity hypothesis holds in zero-pressure-gradient (ZPG) turbulent boundary layers over threedimensional roughness that satisfy the well-characterized condition. The only exception has been reported for a ZPG boundary layer with two-dimensional roughness (Djenidi et al. [17]). When the flow satisfies the wall-similarity hypothesis, the impact of roughness on the outer layer can be presumably accounted for as a mean momentum deficit whose magnitude depends on the roughness geometry. All other effects associated with the surface condition are confined to the roughness sublayer. Yet, Hong et al. [7] and Hong et al.[8] show that even when the profiles of Reynolds stresses satisfy the similarity hypothesis, excess amount of roughness scale turbulence persists in the outer layer, and affects the energy dissipation rate there. The motivation for studying the roughness sublayer is not limited to associating the local turbulence statistics with geometric parameters of the rough surface, but also to elucidate the fundamental processes involved, as well as understand how it affects the near-wall turbulence and its interaction with the outer layer. Answering these questions is essential for the development of appropriate modeling tools. Performing accurate and reliable near-wall flow measurements is challenging. Coexisting high-intensity turbulence and low mean velocity near the wall negatively affects the performance of traditional single-point probes, such as hot-wires, the largely adopted means used to obtain most of the available experimental data, e.g. Perry et al. [18]. Non-invasive optical techniques such as laser Doppler velocimetry (LDV) and particle image velocimetry (PIV) provide noisy or even corrupted data of the very near-wall flow because of reflection from the solid interface. Nevertheless, they have been used to study the roughness sublayer in the low $\delta k^{1}$ regime by Grass [19] and Dancey et al. [20], for instance. To 
(a)

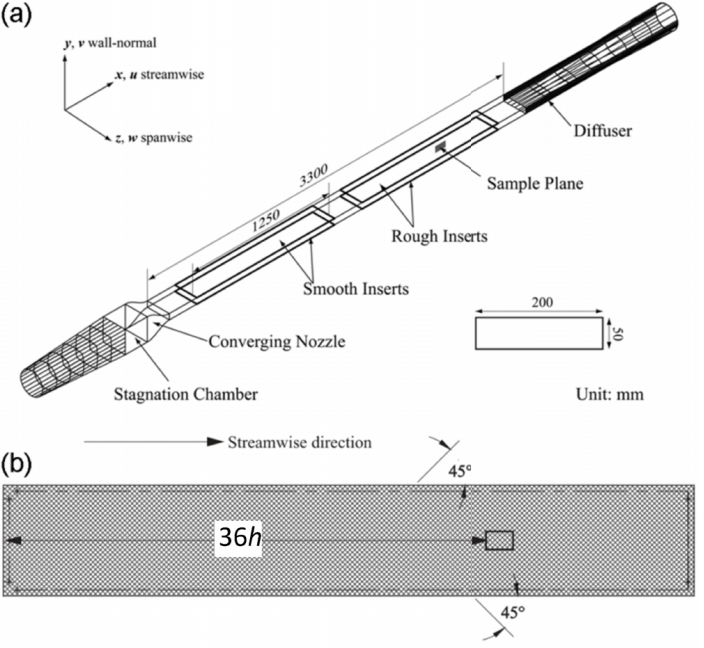

(c)

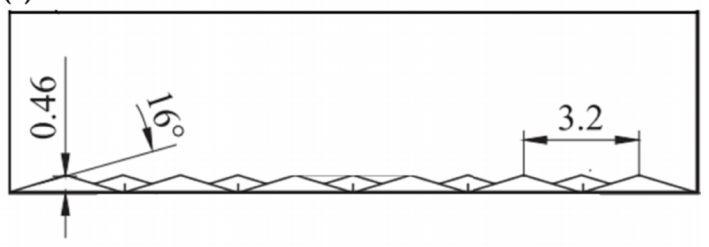

Fig. 1 The bypass channel in the optically indexmatched test facility (a) hosts the rough bottom plate (b). The location of measurement domain is marked by a rectangle and the geometry of the bottom wall roughness is also indicated (c). (a)

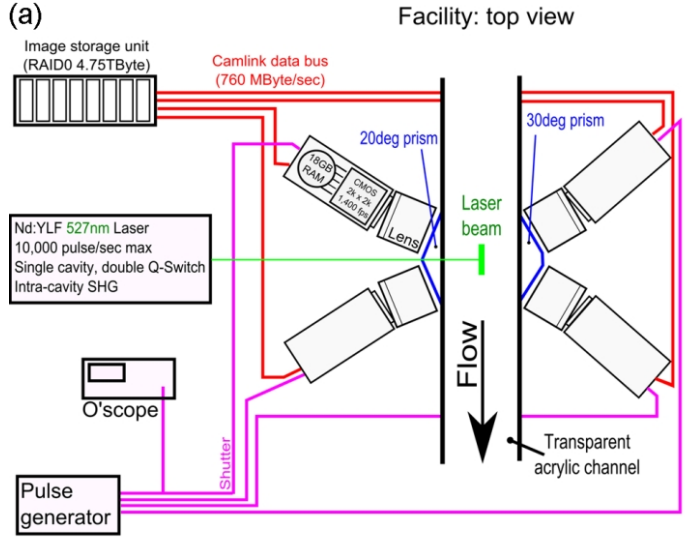

(b)

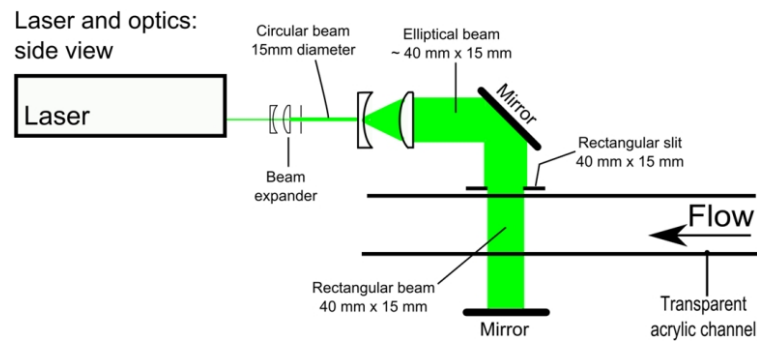

Fig. 2 Top view (a) of the TPIV system mounted around the channel, the laser light is projected using the setup described in (b).

resolve this problem, optical index matching has enabled our group to perform planar PIV, and 3D microscopic holographic PIV measurements in the roughness sublayer (Hong et al. [7], Hong et al.[8], and Talapatra and Katz $[9,10])$.

Since computation speed has been rapidly increasing thorough the years, researcher have become interested in direct numerical simulation (DNS) of turbulence over smooth and rough walls at relatively low, but increasing, wall friction Reynolds numbers. Since roughness substantially increases the number of degrees of freedom of the numerical problem, DNS of rough-wall turbulence has been mostly implemented for two-dimensional roughness at low Reynolds numbers, e.g. at a momentum-thickness-based Reynolds number of $\operatorname{Re}_{\theta}=1400$ in a boundary layer (Lee and Sung [21]) and at $\mathrm{Re}_{\tau}=730-1064$ in channel flows ([Burattini et al. [22]). The flow above three-dimensional roughness is even more complex and, at given computation capability, the achievable Reynolds number is lower, e.g. $\mathrm{Re}_{\tau}=400$ (Bhaganagar et al. [23]). These flows are either in transitionally rough regime - low $k_{\mathrm{s}}^{+}-$or close to the flow over obstacles - low $\delta k^{1}$. To our knowledge, except for the above-mentioned holographic measurements, which are confined to a small volume, and do not extend to the outer layer, the 3D turbulence in the roughness sublayer of a wellcharacterized boundary layer over a dense three-dimensional rough wall has not been resolved yet, either experimentally or computationally.

The objective of this project, for which the present paper provides preliminary results, is to conduct fully-three dimensional, time-resolved investigation of the flow in the outer boundary layer over the same channel that Hong et al. [7], Hong et al. [8], and Talapatra and Katz [9,10] have previously studied. We use time-resolved tomographic PIV (TPIV) in our optical refractive index matched facility to investigate the motion of large scale flow structures that are convected from the rough wall towards the bulk flow. This preliminary step leads toward implementation of a more complex, multi-scale system that combines holographic PIV and TPIV for simultaneous measurements of near-wall and outer boundary layer flow phenomena, respectively.

\section{EXPERIMENTAL SETUP}

The measurements are performed in the bypass channel of our turbomachinery test facility as illustrated in Fig. 1a. The bypass consists of a stagnation chamber, which contains a honeycomb and grids, followed by a converging nozzle, the channel, and a diffuser that delivers the fluid back to the main facility. A detailed description of the setup can be found in Hong et al. [7]. The test section, whose length is $3.3 \mathrm{~m}$ and constant cross section is $203 \times 51 \mathrm{~mm}^{2}$, is entirely made of transparent acrylic to ensure full optical access to any internal region from multiple points of view. Since the cross section aspect ratio is 4:1, three-dimensional flow is expected in the test tunnel as shown by Dean [24], and Monty [25]; nevertheless, since the magnitude of secondary flows is expected to be less than $2-3 \%$ of the centerline, streamwise, 
mean velocity component $\left(U_{0}\right)$ ([Fujita [26], Yokosawa et al. [27]), we use the term "channel" throughout this paper. Two pairs of $1.25 \mathrm{~m}$ long removable acrylic inserts are mounted in the upstream and downstream halves of the test section, as shown in Fig. 1a. The presented data has been recorded with a pair of smooth inserts facing each other symmetrically at top and bottom of the channel at the upstream half, and a pair of rough inserts, which are also symmetrically mounted at the downstream half. As illustrated in Fig. 1b, the measurements have been performed $36 \mathrm{~h}$ downstream of the leading edge of the rough insert, where $h=25.4 \mathrm{~mm}$ is half of the channel height. Early experiments conducted using two-dimensional PIV on various planes inside the channel, for instance by Hong et al. [7], show that the flow there has achieved self similarity. The roughness is composed of closely packed pyramids with height of $0.46 \mathrm{~mm}$ and slope angle of $16^{\circ}$. This geometry falls in the middle of pyramidal shapes tested by Shultz and Flack [5], who, together with Hong et al. [7], show that for the selected roughness, $k_{\mathrm{s}} \sim 1.5 k$. This roughness has two well-defined length scales, and its height $k$ and wavelength $\lambda$ allow spatial sampling of data to be performed while retaining the threedimensional nature of the roughness. The packed pyramids satisfy the "well-characterized" flow conditions (Jiménez [15]), with $h / k=55$ and $k^{+}=64$. A solution of sodium iodide in water flows inside the channel. Its optical refractive index is matched with that of the acrylic channel walls by carefully adjusting the salt concentration $(62-64 \%$, by weight) and temperature. The specific gravity of this solution is 1.8 and its kinematic viscosity $v$ is $1.1 \times 10^{-6}$, i.e. very close to that of water. Further information about the sodium iodide solution can be found in Uzol et al. [28]. The flow is seeded with $11 \mu \mathrm{m}$ hollow-spherical, glass particles that are silver coated to maximize the scattered light intensity. The specific gravity of the tracers is 1.6 , i.e. the particles are slightly buoyant. Our experiments are performed at $\operatorname{Re}_{\mathrm{h}}=U_{0} h v^{1}=40000$, which ias calculated using $U_{0}=1.73 \mathrm{~ms}{ }^{1}$, the mean flow velocity at the channel centerline. This velocity has been inferred using the measured static pressure difference across the inlet contraction of the channel, which has been calibrated using planar PIV prior to the TPIV measurements in the channel centerline up to $\mathrm{Re}_{\mathrm{h}}=78000$.

To obtain three-dimensional snapshots of the flow, a time-resolved tomographic PIV (TPIV) system (Elsinga [29], Elsinga et al. [30]) has been designed and implemented around the channel. Four identical, 2016 $\times 2016$ pixel, high speed CMOS cameras, shown in Fig. 2a, view the same region in the channel through acrylic prisms. We actually record $1200 \times 1200$ pixel images to achieve a data acquisition rate of $3000 \mathrm{frame} / \mathrm{s}$. To record images, we use independently mounted, properly inclined (by the so-called Scheimpflug angle), $60 \mathrm{~mm}$ macro tele-lenses, whose irises are set to f/11 to achieve the desired depth of focus. Images have been acquired using the frame straddling technique (Wing et al. [31]) to obtain 1500 vector fields per second Specifically, the time interval between two consecutive laser pulses that generate an image pair is $150 \mathrm{~s}$, and the time interval between two consecutive image pairs is $t^{*}=0.6667 \mathrm{~ms}$. In order to achieve a laser pulse rate of 3000 pulses per second, while still illuminating the particles with enough energy for detection, we have opted to use a high speed Nd:YLF laser (527 nm). As shown in Fig. 2b, the $32 \mathrm{~mJ} /$ pulse laser beam is collimated, expanded and projected inside the channel through a slit to obtain a thick collimated light sheet. To increase the illumination intensity, the laser sheet is reflected back into the channel through the bottom acrylic plate using a dielectric flat mirror.

The calibration is performed using a vertical 2D target, consisting of a $0.1 \mathrm{~mm}$ black metal foil sandwiched between two transparent and refractive index matched acrylic plates. Consequently, only the foil is visible in the calibration images. The thickness of this foil has been taken into account during calibrations. A Cartesian pattern of dots is generated on the metal foil by laser-cutting. The diameter of the each dot is $1 \mathrm{~mm}$, and the spacing between them is $2 \mathrm{~mm}$, center to center. The metal sheet and associated acrylic plates are mounted on a translation stage that ensures precise positioning of the calibration target, as it is translated with $0.01 \mathrm{~mm}$ resolution in the spanwise direction to cover the entire domain of interest. During calibration, the target is translated from $z=-7.5 \mathrm{~mm}$, i.e. $2.343 \lambda$, where $\lambda=3.2 \mathrm{~mm}$ is the roughness wavelength (peak to peak distance in the streamwise and spanwise directions), to $z=+7.5 \mathrm{~mm}$ with steps of $z=1.5 \mathrm{~mm}(0.469 \lambda)$. Therefore, the entire calibration consists of 11 sets of images recorded by the four cameras using diffused ambient light. The calibration images have been imported into the La Vision DaVis software for the initial mapping of domain geometry, and for calculating of associated mapping coefficients. Subsequently, single image volume self-calibration (Michaelis and Wolf [32]) is performed to further improve the accuracy of the calibrations.

\section{PARTICLE DISTRIBUTION RECONSTRUCTION AND CROSS-CORRELATION}

Images acquired during the experiment are pre-processed prior to tomographic reconstruction of the three-dimensional particle distribution to enhance the signal-to-noise ratio of raw data, and improve the speed and reliability of the reconstruction. The image processing routine is identical for all the cameras. First, the time sequence of images is averaged and the average is subtracted from each and every frame in the sequence. This step improves the image contrast by eliminating bias noise in the background. Subsequently, images are processed using the Modified Histogram Equalization (MHE) software (Roth and Katz [34]), which further enhances the contrast between tracers and the background. MHE automatically equalizes the average luminance of the images acquired by the 4 cameras because the algorithm has been implemented while setting the same parameters for all the images. Tomographic reconstruction of the particle distributions has been performed using the Multiplicative Algebraic Reconstruction Technique (MART) (Elsinga, [29]). The three-dimensional particles traces have been Gaussian filtered before each of the first four 
iterations, but the last two rounds have been implemented without filtering to minimize the reconstructed particle size, and to reduce the uncertainty in the position of its centroid [Dirk Michaelis, La Vision, private communication]. The MART algorithm is recursive and contains a relaxation parameter $\mu$ (Elsinga, [29]) whose value is selected as a compromise between speed, which improves with increasing values, and accuracy, which improves with decreasing $\mu$. Typically, $\mu=1$ is a good compromise, but we have also performed MART reconstruction using $\mu=0.2$, as suggested in Elsinga [29], and $\mu=0.5$, which has proven to be the optimal value for our data. Specifically, $\mu=0.2$ and 0.5 have generated very similar smooth vector fields, but the latter value also results in faster reconstruction.

Following the reconstructions, multi-pass cross-correlations of the 3D particle distribution pairs have provided 195 three-dimensional velocity fields. The last step of the cross-correlation process is performed using $64 \times 64 \times 64$ voxel interrogation volumes, which, with $75 \%$ overlap, provides a final velocity vector spacing of $0.588 \times 0.588 \times 0.588 \mathrm{~mm}$, i.e. $1.278 \mathrm{k}$. The dimensions of velocity field domain are $l_{\mathrm{x}} \times l_{\mathrm{y}} \times l_{\mathrm{z}}=40.6 \times 44.1 \times 17.4 \mathrm{~mm}^{3}$, where $l_{\mathrm{x}}, l_{\mathrm{y}}, l_{\mathrm{z}}$ are, respectively, the streamwise, wall-normal, and spanwise directions (Fig. 1a). The vector fields in the time series have been smoothed temporally as well, using a three fields sliding average.

\section{RESULTS}

The results presented in this paper focus on the evolution and transport of flow structures based on examination of the initial set 195 three-dimensional velocity distributions. Due to the relatively coarse velocity vector spacing, $1.278 k$, we cannot describe the flow between roughness elements, as done in Talapatra and Katz [9,10], and we limit our analysis to the flow immediately above the roughness. Figure 3 shows four sample instantaneous snapshots of the flow at different instants, $t=55 t^{*}, t=68 t^{*}, t=75 t^{*}$, and $t=80 t^{*}$, where $t^{*}=0.6667 \mathrm{~ms}$ is the time interval between two consecutive vector fields in the time series. The inflow and outflow sections are located at $x / \lambda=0$ and $x / \lambda=12.87$, respectively, and the measurement domain is bounded vertically between $y / k=0$, the top of the roughness elements, and $y / k=95.93$, corresponding to $y / h=1.74,86.5 \%$ of the total channel height. In the spanwise direction, the domain is confined between $z / \lambda= \pm 2.76$, and the location of roughness element peaks on the $y / k=0$ plane is indicated in Fig. 3 by white dots. The distributions of instantaneous velocity magnitudes normalized by the mean centerline velocity in the inflow and outflow wall-normal-spanwise $(y-z)$ planes are color-coded using the scale provided. In addition, we also show the velocity for a $(y-z)$ plane located in the middle of the sample. The velocity components, $u, v$ and $w$ correspond to the streamwise, wall-normal, and spanwise directions respectively. Since a sequence of 195 vector fields recorded at 1500 field per second does not constitute an ensemble, we cannot use the presently available data to obtain ensemble averaged flow or turbulence quantities. Nevertheless, the velocity peaks at $y / h=\sim 1$, where $\left(u^{2}+v^{2}+w^{2}\right)^{0.5} / U_{0} \sim 1.05$, and the velocity magnitude decreases as the wall is approached, both consistent with expectations. However, because of the relatively coarse vector spacing, the vectors located closest to the wall still have a magnitude of $\left(u^{2}+v^{2}+w^{2}\right)^{0.5} \backslash U_{0} \sim 0.55$. Figure 3 also shows iso-surfaces of normalized vorticity magnitude, selected as $=\left(\omega_{\mathrm{x}}{ }^{2}+\omega_{\mathrm{y}}{ }^{2}+\omega_{\mathrm{z}}{ }^{2}\right)^{0.5} \quad\left(k \backslash U_{0}\right)=0.452$. Transport of near-wall high vorticity regions is evident in the time sequence, especially near both boundaries, e.g. the vortical structure indicated with $\mathrm{A}$ is advected in the streamwise direction by $\quad x / \lambda \sim 7$, as its shape changes substantially between frames. The vorticity iso-surfaces also show other small scale vortices transported by the bulk flow.

Using time resolved planar PIV and holographic PIV, Hong et al. [7,8] and Talapatra and Katz [9,10], respectively, performed detailed measurements using the same setup, but at higher $\mathrm{Re}_{\mathrm{h}}$. Their observations have led them to propose a model for characteristic flow structures causing rapid lifting of vortices away from the wall consisting of the previously mentioned interacting U-shaped vortices. They are illustrated qualitatively in Figure 4d. Consequently, we use the present TPIV data to corroborate (or disprove) their model. Figure 4 shows samples of the lowest portion of the investigated domain together with the location of roughness element peaks, each showing planar distribution of vertical velocity superimposed with black contours of streamwise vorticity, with positive and negative vorticity contours indicated by continuous and dashed lines, respectively. At $x / \lambda=5$ and $t=77 t^{*}$, Fig. 4a shows a pair of counter-rotating vortices with a relatively intense negative vertical velocity between them. This downward flow is nearly aligned with the spanwise location of a pyramid peak. As the $(y-z)$ plane slicing the sample volume is shifted downstream to $x / \lambda=5.6$, but still for the same instant, the velocity and vorticity distributions change significantly. Most notably, the two region positive $\omega_{\mathrm{x}}$ highlighted in Fig. $4 \mathrm{a}$ migrate away from the wall, and merge in Fig. 4b. At the same time, the marked negative $\omega_{\mathrm{x}}$ area becomes more intense, but does not change its elevation appreciably, and in fact, it remains approximately aligned with the roughness trough between pyramid ridgelines. In addition, a newly generated positive $\omega_{\mathrm{x}}$ region appears at $z / \lambda=1.9$ and $y / k=2.5$, creating a near-wall counter-rotating pair with the negative vorticity to the left of it. As the $(y-z)$ plane is shifted to $x / \lambda=7.5$, the same near wall pair of opposite $\omega_{\mathrm{x}}$ rises to $y / k=7.5$, i.e. they rise at an angle of $\sim 21^{\circ}$ with the streamwise direction, and there is a region with high positive vertical velocity (an ejection) between them. Note that both quasi-streamwise vortices are roughly aligned with the roughness troughs. The holographic results of Talapatra and Katz $[9,10]$ for $\mathrm{Re}_{\mathrm{h}}=64500$ also show regions of strong vertical advection in the $0<y / k<4$ interval, which are centered between rapidly rising quasi streamwise vortices originated from neighboring pyramids. In their case, the (statistically determined) inclination angle of structures is much higher, reaching $54^{\circ}-63^{\circ}$. This discrepancy might be related to differences in spatial resolution of measurements (theirs is $0.13 k$ ), or to differences in the elevation of measurements. The quasi-streamwise vortices first observed by Talapatra and Katz [9,10] are measured in the $0<y / k<4$ range, while vortices described in this paper are situated much further away from the wall, at 

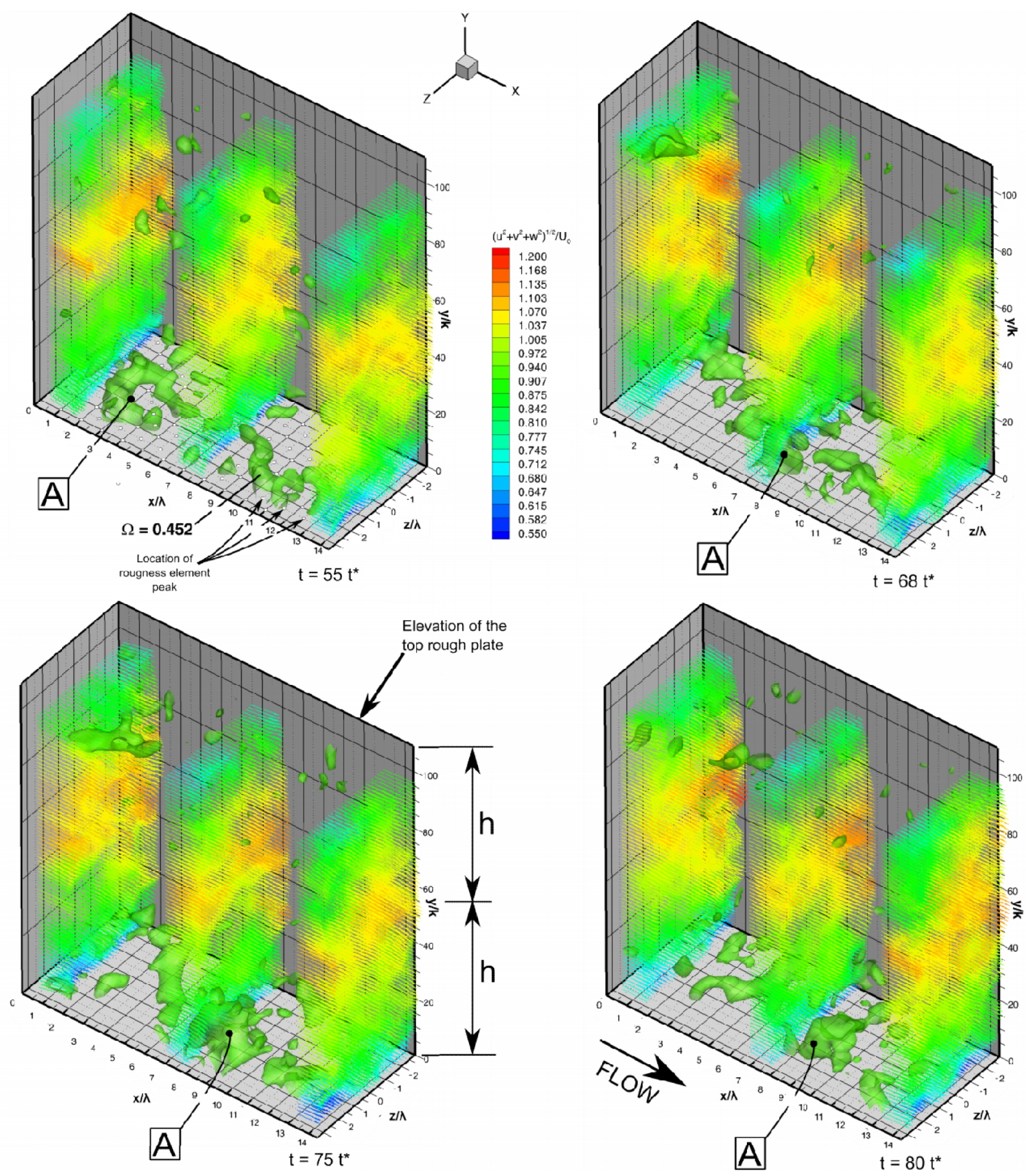

Fig. 3 Snapshots of the instantaneous flow in the channel at four instants $55 t^{\star}, 68 t^{\star}, 75 t^{\star}$, and $80 t^{\star}$. Velocity vectors are shown at the inflow $(x / k=0)$, middle $(x / k=44.45)$, and outflow $(x / k=88.90)$ sections and colored using the normalized velocity magnitude. Iso-surfaces of the normalized vorticity magnitude $=0.425$ allow the structure indicated by $A$ to be followed in time. Location of roughness element peaks is marked by white dots in the top left subfigure.

$2.5<y / k<7.5$. It is plausible that the two data sets show different phases in the development of the same structures. Since streamwise advection quickly increases with distance from the wall, it would inherently reduce the inclination angle of rising structures. This issue will be investigated in the near future based on statistical analysis of the present results.

Following the work of $\mathrm{Wu}$ and Christensen [4] we opt to investigate the advection of low speed streamwise streaks at three elevations from the rough wall, i.e. $y / k=1, y / k=2$, and $y / k=4$. Figure 5a shows a time series of the same $(x-z)$ "slice" of the flow within the time interval $55 t^{*}<t<90 t^{*}$. Here, contour lines of wall-normal vorticity, with dashed lines indicating negative values, are superimposed on color contours of the normalized streamwise velocity $u / U_{0}$. Regions of low streamwise velocity $\left(u / U_{0} \sim 0.55\right)$ with width of about $1 \lambda$ are evident. Once formed, they appear to be 

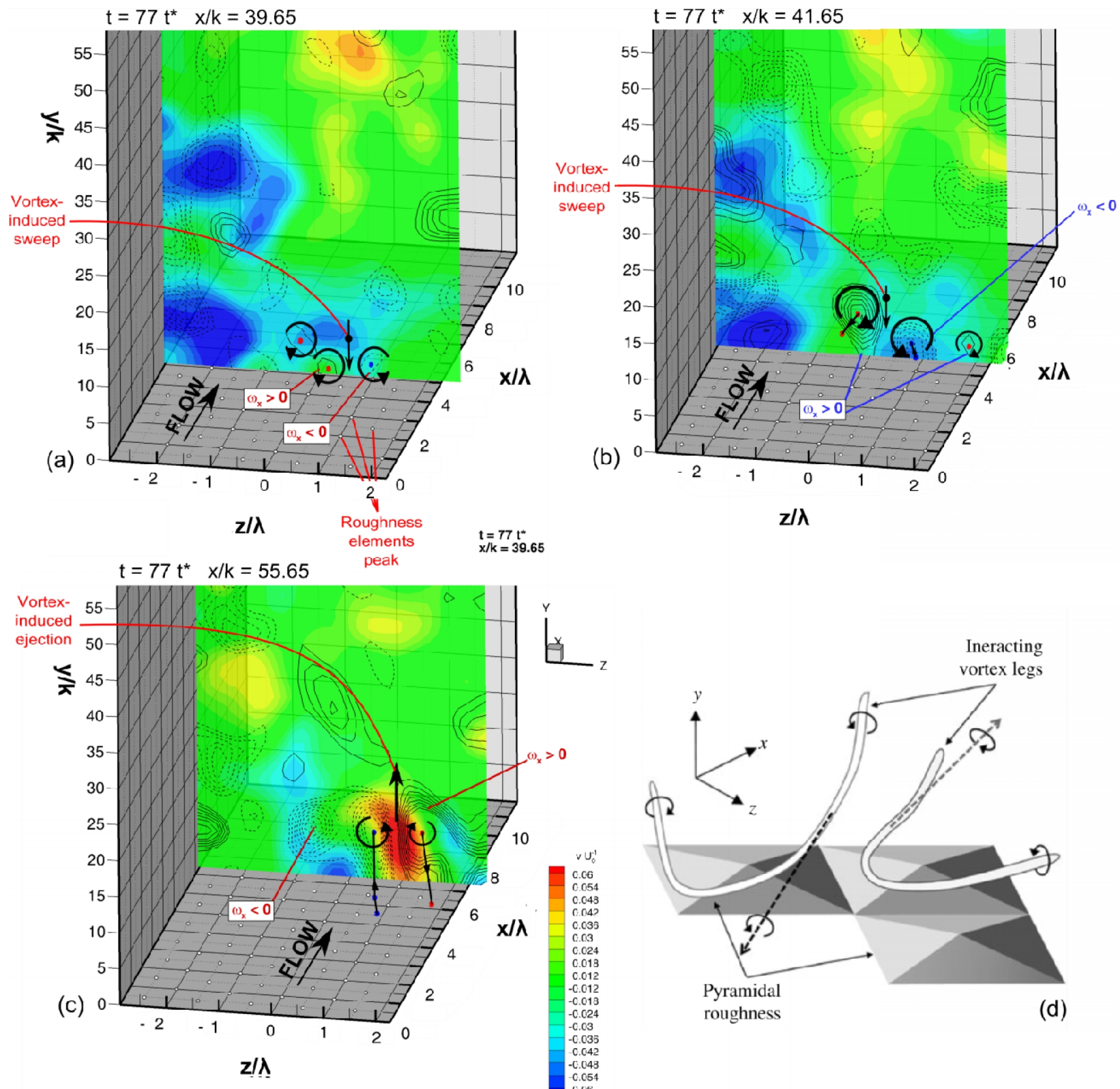

Fig. 4 A single velocity snapshot captured at instant $77 t^{\star}$ is sliced at three different streamwise locations indicated in figure. Center and swirl associated with some of the newborn flow structures are tracked as the color contoured plane moves downstream. Wall-normal velocity and streamwise vorticity components are indicated on the plane by colors and contour lines respectively. Dashed lines indicate negative vorticity component. Location of the roughness elements peak is marked by white dots. A sketch (d) describing a proposed model (Talapatra and Katz [10]) for the observed phenomenon is also included.

"channeled" between consecutive pyramid ridgelines. Note that the location of selected roughness peaks in Fig. 5a and Fig. $5 \mathrm{~b}$ is highlighted with white dots for clarity. Since the maps shown are separated by the same time intervals, $5 t^{*}$, one can infer the rate of displacement of selected streaks by connecting them. We obtain an approximate displacement rate of $1.23 \mathrm{~ms}^{1}\left(0.71 U_{0}\right)$ for the flow presented in Fig. 5a, and $0.55 \mathrm{~ms}^{1}\left(0.32 U_{0}\right)$ for the series shown in Fig. $5 \mathrm{~b}$. These visual approximations will be followed by correlation based statistical of the velocity fields. Presently, the analysis of the evolution of the vertical structures presented in Fig. 5a and Fig. 5b is performed by tracking the motion of high $\omega_{\mathrm{y}}$ regions at every time step. Interestingly, some of the $\omega_{\mathrm{y}}$ peaks appear to be latched to certain position in the $(x-z)$ plane, and seem to be convected downstream intermittently, at a very low velocity. Because the downstream advection never ceases, the perception that the vortices do not move is an illusion caused by continuous production of $\omega_{\mathrm{y}}$ in certain regions, presumably due to the interaction of the flow with the stationary roughness element. Although we mark and connect the latched $\omega_{\mathrm{y}}$ peaks in subsequent realizations, they do not represent region of the same fluid. As the elevation of the $(x-z)$ plane is increased, while keeping the same time interval, $55 t^{*}<t<90 t^{*}$, the appearance of low speed streaks changes. At $y / k=2$, the streamwise extents and width of streaks decrease, and the "channeling" along roughness ridgelines is less evident. However, as shown in Figure $6 a$, the fixed regions of $\omega_{\mathrm{y}}$ peaks are still evident, and 


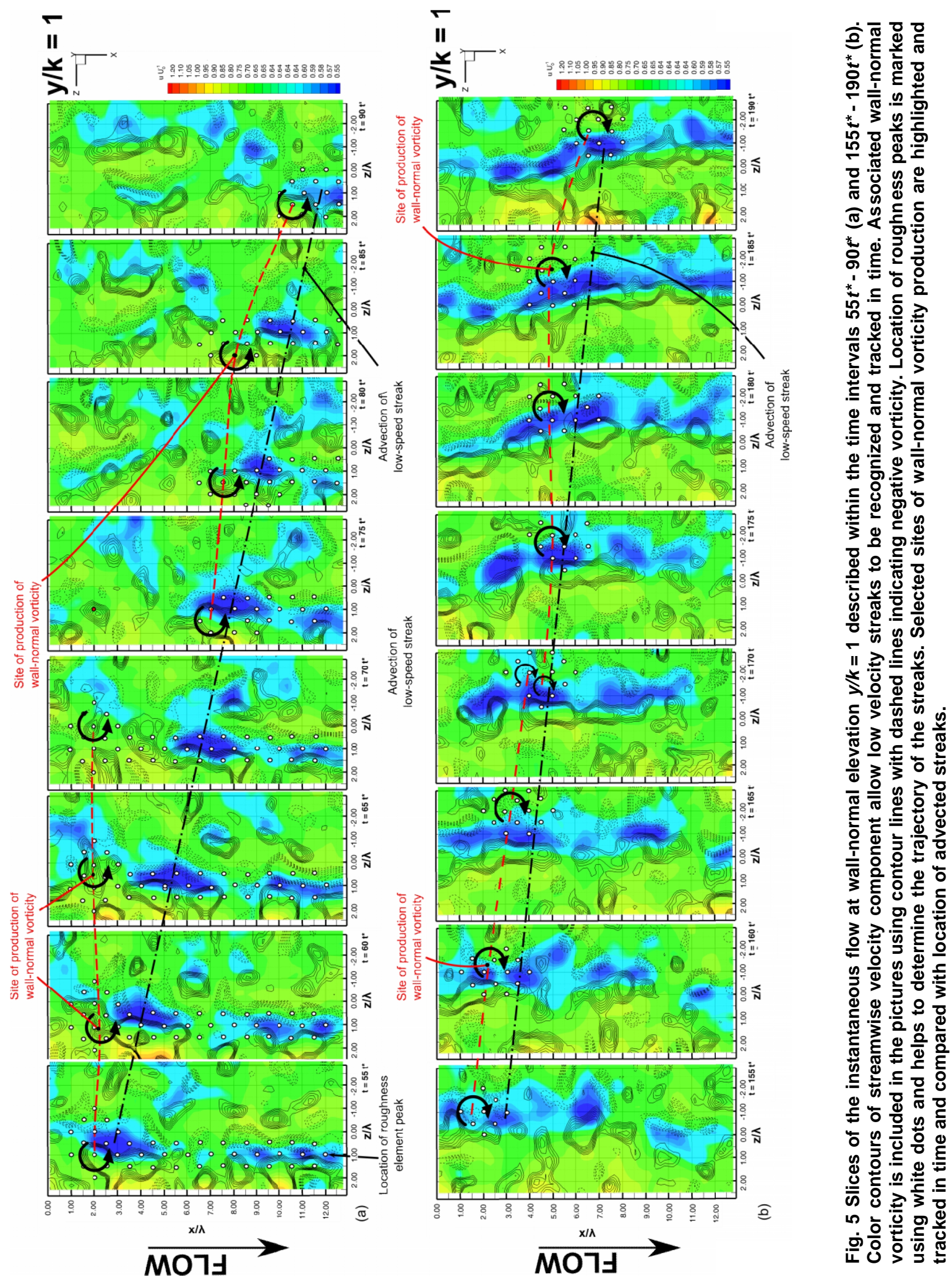




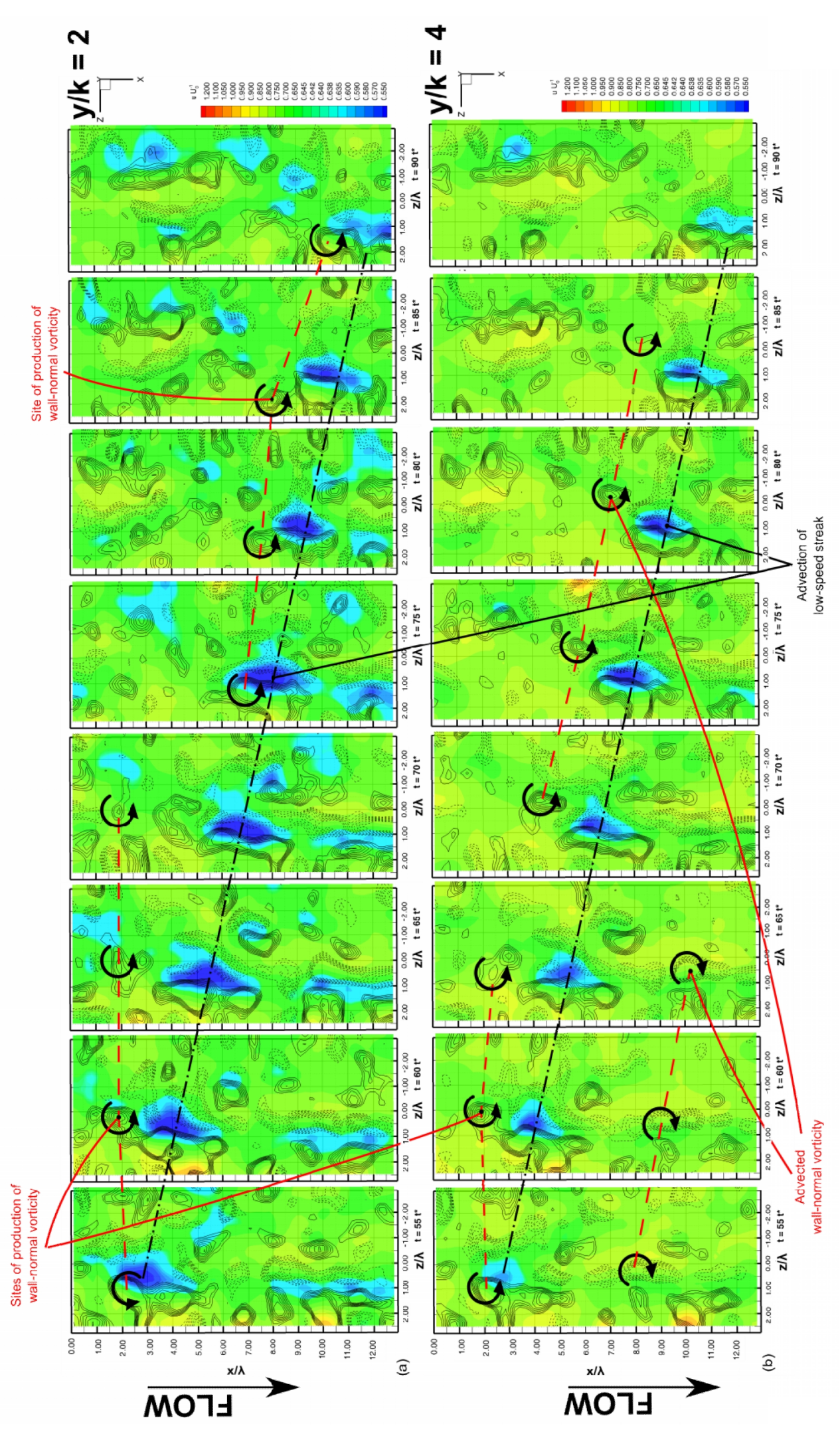

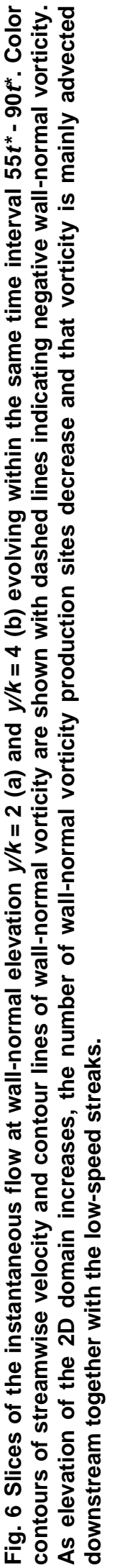


they are situated approximately at the same location as those shown in Figure 5a. The trends for $y / k=4$ could be compared to those of $\mathrm{Wu}$ and Christensen [4] who provide data on the flow and turbulence, including width of low speed streaks, based on planar and stereo-PIV measurements above irregular roughness for the same normalized elevation. The width of their low velocity streak, obtained based on spatial cross-correlation of the streamwise velocity fluctuation, is $z / k \sim 11$, while the width of the streak presented in Figure $6 \mathrm{~b}$ is only $z / k \sim 7$. The discrepancy might not mean much since, at the present time, our results are based on a very limited database (one case), whereas Wu and Christensen's [4] conclusions are based on statistical analysis of a large database. The resolution of our measurements might also affect our conclusions. To address this question, we intend to compare the present PIV results to those obtained based on much higher resolution planar data obtained previously by Hong et al. [8] for the same setup. Also, note that the latched/fixed sites of $\omega_{\mathrm{y}}$ peaks at $y / k=4$ are much less evident than those observed lower elevations. Figure $6 \mathrm{~b}$ shows that while the wall-normal vorticity peak is still fixed near $x / \lambda=2$ and $1<z / \lambda<0$, the $\omega_{\mathrm{y}}$ peaks elsewhere are mainly advected with the same rate of displacement of the low $u / U_{0}$ streaks.

\section{CONCLUSIONS}

This paper describes early phases of our effort to elucidate the 3D flow structure in the inner part of a rough-wall turbulent channel flow based on time resolved tomographic particle image velocimetry (TPIV) measurements. This work is inspired by earlier experiments (along with numerous other studies) involving applications of planar PIV and 3D holographic PIV to characterize the same flow. The planar measurements have indicated that 3D data are essential for resolving the flow. The holographic data has a much higher resolution than the present TPIV measurements, but the dimensions of the sample volume are smaller than one roughness wavelength, preventing us from examining interactions of the near wall flow structures with much larger eddies residing in the outer layer, and the spatio-temporal evolution of these structures. Ultimately, our intention is perform multi-scale measurements, namely combine the relatively coarse TPIV data with simultaneously obtained fine scale holographic measurements. The present 3D measurements cover the entire boundary layer, including the roughness sublayer, requiring the TPIV sample volume to be located very close to the rough wall. To facilitate such measurement, we match the optical refractive index of the channel acrylic rough walls with that of the fluid, a concentrated solution of sodium iodide in water. The roughness consists of staggered pyramids whose $h / k=55$ and $k^{+}=64$ satisfy the well-characterized condition. Experiments are performed at Reynolds number of 40000 , based on the channel half-height and mean centerline velocity. Acquiring data at 3000 frames per second, and utilizing frame straddling, enables us to and generate 1500 three-dimensional vector fields per second, allowing us to observe and track flow structures in space and time. Preliminary results presented in this paper are limited to 195 instantaneous velocity fields. Consistent with measurements performed on the same channel using holographic PIV, the present results confirm that U-shaped vortices wrap around low speed region that extend above the roughness element peaks. As quasi-streamwise "legs" of vortices generated above neighboring pyramids interact, they induce powerful ejection events that lift these structures away from the wall and tilt the "legs" at steep angles. The spatial extent and migration rate of low streamwise velocity streaks have been evaluated, for now based on a few selected instantaneous snapshots of the flow. These anecdotal results will be refined in the near future using spatial correlations of the fluctuating velocity distributions. With increasing distance from the rough wall, the width of streaks decreases, but their displacement rate is not affected significantly by elevation. Planar distribution and advection trends of wall normal vorticity in $(x-z)$ planes (parallel to the rough wall) vary with distance from the wall. At $1<y / k<4$, the signature of roughness elements is evident as fixed sites of wall-normal vorticity, while at $y / k=4$, some sites are still discernible, but elsewhere the wall-normal vorticity is mainly advected at the same rate of the low speed streaks.

\section{ACKNOWLEDGEMENTS}

The rough wall studies have been funded by ONR; R. Joslin is the program officer. Development of the high speed tomographic PIV system has been funded by NSF MRI grant No. 0923391. We would like to thank Yury Ronzhes and S. King for the help provided in implementing and maintaining the facility.

\section{NOMENCLATURE}

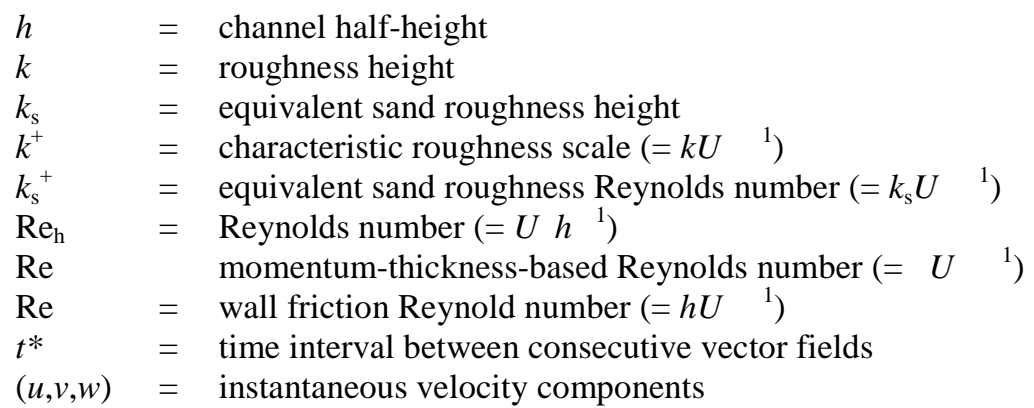


$U_{0} \quad=\quad$ mean, centerline, streamwise velocity component

$\begin{array}{lll}\text { Greek } & \\ \delta & = & \text { boundary layer thickness } \\ \delta^{+} & = & \text {characteristic boundary layer thickness } \quad\left(=\delta U_{\tau} v^{1}\right) \\ \lambda & = & \text { roughness wavelenght } \\ \mu & = & \text { MART relaxation parameter } \\ \omega & = & \text { vorticity } \\ & = & \text { normalized vorticity magnitude }\end{array}$

\section{REFERENCES}

[1] Towsend A. A., The Structure of Turbulent Shear Flow.

[2] Raupach, M. R., Antonia, R. A. \& Rajagopalan, S. 1991 Rough-wall turbulent boundary layers. Appl. Mech. Rev. 44, 1-25.

[3] Shockling M. a., Allen J. J., and Smits A. J., 2006, "Roughness effects in turbulent pipe flow," Journal of Fluid Mechanics, 564, p. 267.

[4] Wu Y., and Christensen K. T., 2007, "Outer-layer similarity in the presence of a practical rough-wall topography," Physics of Fluids, 19(8), p. 085108.

[5] Schultz M. P., and Flack K. a., 2003, "Turbulent Boundary Layers Over Surfaces Smoothed by Sanding," Journal of Fluids Engineering, 125(5), p. 863.

[6] Flack K. A., Schultz M. P., and Shapiro T. A., 2005, "Experimental support for Townsend's Reynolds number similarity hypothesis on rough walls," Physics of Fluids, 17(3), p. 035102.

[7] Hong J., Katz J., and Schultz M. P., 2011, "Near-wall turbulence statistics and flow structures over three-dimensional roughness in a turbulent channel flow," Journal of Fluid Mechanics, 667, pp. 1-37.

[8] Hong J., Katz J., Meneveau C., and Schultz M. P., 2012, "Coherent structures and associated subgrid-scale energy transfer in a rough-wall turbulent channel flow," Journal of Fluid Mechanics, 712, pp. 92-128.

[9] S. Talapatra and J. Katz, 2013, Three-dimensional velocity measurements in a roughness sublayer using microscopic digital inline holography and optical index matching, Meas. Sci. Technol., (24), 024004, doi:10.1088/0957-0233/24/2/0

[10] Talapatra S., and Katz J., 2012, "Coherent structures in the inner part of a rough-wall channel flow resolved using holographic PIV," Journal of Fluid Mechanics, 711, pp. 161-170.

[11] Krogstadt P.-Å., and Antonia R. A., 1999, "Surface roughness effects in turbulent boundary layers," Experiments in Fluids, 27(5), pp. 450-460.

[12] Tachie M. F., Bergstrom D. J., and Balachandar R., 2000, "Rough Wall Turbulent Boundary Layers in Shallow Open Channel Flow," Journal of Fluids Engineering, 122, pp. 533-541.

[13] Shah M. K., and Tachie M. F., 2009, "PIV investigation of flow over a transverse square rib in pressure gradients," Journal of Turbulence, 10(January 2013), p. N39.

[14] Keirsbulck L., Labraga L., Mazouz a., and Tournier C., 2002, "Surface Roughness Effects on Turbulent Boundary Layer Structures," Journal of Fluids Engineering, 124(1), p. 127.

[15] Jimenez J., 2004, “Turbulent Flows Over Rough Walls,” Annual Review of Fluid Mechanics, 36(1), pp. $173-196$.

[16] Volino R. J., Schultz M. P., and Flack K. a., 2009, "Turbulence structure in a boundary layer with two-dimensional roughness," Journal of Fluid Mechanics, 635, p. 75.

[17] Djenidi L., Antonia R. a., Amielh M., and Anselmet F., 2007, “A turbulent boundary layer over a two-dimensional rough wall," Experiments in Fluids, 44(1), pp. 37-47.

[18] Perry E., Lim K. L., and Henbest M., 1987, "An experimental study of the turbulence structure in smooth- and rough-wall boundary layers," Journal of Fluid Mechanics, 177, pp. 437-466.

[19] Grass a. J., 2006, "Structural features of turbulent flow over smooth and rough boundaries," Journal of Fluid Mechanics, 50(02), p. 233.

[20] Dancey C. L., Balakrishnan M., Diplas P., and Papanicolaou A. N., 2000, "The spatial inhomogeneity of turbulence above a fully rough , packed bed in open channel flow," Experiments in Fluids, 29, pp. 402-410.

[21] Lee S.-H., and Sung H. J., 2007, "Direct numerical simulation of the turbulent boundary layer over a rod-roughened wall," Journal of Fluid Mechanics, 584, p. 125.

[22] Burattini P., Leonardi S., Orlandi P., and Antonia R. a., 2008, "Comparison between experiments and direct numerical simulations in a channel flow with roughness on one wall," Journal of Fluid Mechanics, 600, pp. 403-426.

[23] Bhaganagar K., Kim J., and Coleman G., 2004, "Effect of Roughness on Wall-Bounded Turbulence," Flow, turbulence and combustion, 72(2-4), pp. 463-492.

[24] Dean, 1978, "Reynolds Number Dependence of Skin Friction and Other Bulk Flow Variables in Two-Dimensional Rectangular Duct Flow," Journal of Fluids Engineering, 100, pp. 215-223.

[25] Monty, 2005, "Developments In Smooth Wall Turbulent Duct Flows," The University of Melbourne.

[26] Fujita H., Yokosawa H., and Hirota M., 1989, "Secondary Flow of the Second Kind in Rectangular Ducts with One Rough Wall," Experimental Thermal and Fluid Science, 2, pp. 72-80.

[27] Yokosawa H., Fujita H., Hirota M., and Iwata S., 1989, "Measurement of turbulent flow in a square duct with roughened walls on two opposite sides," International Journal of Heat and Fluid Flow, 10(2), pp. 125-130.

[28] Uzol O. U., Chow Y.-C. C., Katz J. K., and Meneveau C. M., 2002, "Unobstructed particle image velocimetry measurements within an axial turbo-pump using liquid and blades with matched refractive indices," Experiments in Fluids, 33(6), pp. 909-919.

[29] Elsinga G. E., 2006, “Tomographic particle image velocimetry,” Experiments in Fluids, 41(6), pp. 933-947. 
[30] Elsinga, Wieneke, Scarano, and Schroder, 2008, “Tomographic 3D-PIV and Applications,” Topics Appl. Physics, 112, pp. 103125.

[31] Wing T Lai, Daniel C Bjorkquist, Martin P Abbott and Amir A Naqwi, 1998 , Video systems for PIV recording, Meas. Sci. Technol. 9297 doi:10.1088/0957-0233/9/3/002

[32] D. Michaelis and C. C. Wolf, 2011, Vibration Compensation for Tomographic PIV using Single Image Volume Self Calibration, Proceedings of the 9th international symposium on particle image velocimetry, Kobe, Japan, July 21-23.

[34] Roth G. I., and Katz J., 2001, "Five techniques for increasing the speed and accuracy of PIV interrogation," Measurement Science and Technology, 12(3), pp. 238-245. 